\title{
SÍNDROME DE OGILVIE POSHISTERECTOMÍA: REPORTE DE UN CASO Y REVISIÓN DE LA LITERATURA
}

\author{
Ogilvie's syndrome post hysterectomy: a case report \\ and literature review \\ Jairo Hernández-Pinzón, M.D. *, Marcos Castillo-Zamora, M.D.**, \\ Carolina Rodríguez, M.D.*** \\ Recibido: febrero 19/09 - Aceptado: agosto 31/09
}

\section{RESUMEN}

Objetivo: presentar el estudio y el tratamiento que se le realizó a una mujer embarazada que presentó Síndrome de Ogilvie (SO) como complicación de una histerectomía obstétrica, así como describir la epidemiología, la fisiopatología, los hallazgos clínicos después de los procedimientos gineco-obstétricos y el tratamiento de esta patología mediante una revisión de la literatura.

Presentación del caso: mujer de 28 años de edad con embarazo de 38 semanas de gestación quien después de parto vaginal eutócico sufrió atonía uterina. Debido a la falta de mejoría con el manejo médico, se le realizó una histerectomía abdominal subtotal, presentando picos febriles, intolerancia a la vía oral, taquicardia, distensión e hipertimpanismo abdominal con dolor a la palpación superficial y profunda en hemiabdomen derecho y peristaltismo disminuido durante el posoperatorio. La radiografía de abdomen evidenció un cuadro obstructivo con

\footnotetext{
* Médico General, Servicio de Control Prenatal, Hospital Comunal Malvinas. Regional Representative of Latin-America S.N.O, The Network: TUFH (Towards Unity for Health). Grupo de Investigación: Salud Sexual y Procreativa de la Mujer, Facultad de Medicina, Universidad de la Sabana, Clínica Universitaria Teletón, Chía (Colombia). Correo electrónico: jairohernandez1@hotmail.com

** Especialista en Ginecología y Obstetricia. Profesor Auxiliar, Universidad de La Sabana, Chía (Colombia).

*** Especialista en Cirugía General. Profesora Auxiliar, Universidad de La Sabana, Chía (Colombia).
}

origen probable a nivel del intestino delgado, por lo que se le practicó una laparotomía exploratoria donde se le encontró peritonitis fecal y perforación colónica a nivel de la válvula ileocecal, sin evidencia de obstrucción mecánica.

Discusión: el SO se caracteriza por la dilatación masiva y progresiva del colon en ausencia de una obstrucción mecánica. Se desarrolla principalmente en pacientes hospitalizadas con graves condiciones médico-quirúrgicas, pero también se presenta frecuentemente después de procedimientos gineco-obstétricos.

Debido a que este síndrome se ha visto asociado con una alta morbimortalidad, es necesario que el gineco-obstetra lo tenga en cuenta como diagnóstico diferencial y esté atento a su presentación como complicación de cirugías obstétricas o ginecológicas.

Palabras clave: Síndrome de Ogilvie, pseudoobstrucción colónica aguda, complicación, histerectomía, cesárea.

\section{SUMMARY}

Objective: presenting the workup and management of a pregnant woman who presented Ogilvie's syndrome as a complication of an obstetric hysterectomy and describing the epidemiology, physiopathology and clinical findings following 
gynecobstetric procedure and treatment of this pathology by means of a literature review.

Case report: the case of a 28 -year-old woman is presented; the patient presented uterine atony following normal vaginal delivery after 38 weeks' pregnancy. A subtotal abdominal hysterectomy was made due to lack of improvement with medical management. The patient presented fever, intolerance to oral route, tachycardia, distended and hypertympanic abdomen, pain in response to surface and deep palpation of the right hemi-abdomen and decreased peristalsis during postoperative period. The abdominal radiograph revealed obstruction of the small intestine; exploratory laparotomy was thus carried out, showing foetal peritonitis and perforation of the colon at the ileocecal valve without evidence of mechanical obstruction.

Discussion: Ogilvie's syndrome is characterised by massive and progressive dilatation of the colon in the absence of small bowel obstruction. The syndrome mainly develops in hospitalised patients suffering from severe medical conditions; nonetheless, it also occurs after gynecobstetric procedures. Because this syndrome is associated with high rates of morbidity and mortality, the gynecobstetrician must recognise this syndrome as being a possible complication of gynecobstetric procedure.

Key words: Ogilvie's syndrome, acute colonic pseudo-obstruction, complication, hysterectomy, caesarean section.

\section{INTRODUCCIÓN}

El Síndrome de Ogilvie (SO) fue descrito por primera vez por Sir Heneage Ogilvie en el año 1948 en el British Medical Journal. ${ }^{1}$ Posteriormente, en 1958, Dudley y sus colegas utilizaron el término pseudoobstrucción para describir el aspecto clínico de una obstrucción mecánica sin evidencia de alguna enfermedad orgánica durante la laparotomía. ${ }^{2}$ Este síndrome es una patología poco frecuente, de la cual aún se desconoce con exactitud su epidemiología y su fisiopatología. Aún así, la literatura disponible describe la relación existente entre algunos procedimientos ginecoobstétricos y el síndrome, por lo cual es importante que el especialista en obstetricia y ginecología conozca los aspectos más relevantes de esta entidad.

Por esta razón, el objetivo de la presentación del caso es hacer una revisión global de los datos disponibles sobre la epidemiología, la fisiopatología, los hallazgos clínicos después de procedimientos gineco-obstétricos, así como el tratamiento para el Síndrome de Ogilvie.

\section{DESCRIPCIÓN DEL CASO CLÍNICO}

Mujer de 28 años de edad que consultó la Clínica Universitaria Teletón (CUT) con embarazo de 38 semanas en trabajo de parto, fase expulsiva. La CUT es un hospital general para pacientes del seguro social. Se atendió parto vaginal eutócico, obteniéndose un recién nacido vivo de sexo masculino de 3.100 g APGAR 9/10. A los 20 minutos posparto, la paciente presentó atonía uterina con inestabilidad hemodinámica sin respuesta adecuada al manejo farmacológico y médico, por lo cual se decidió practicarle una histerectomía abdominal subtotal. Durante el procedimiento quirúrgico, la paciente recibió 4 unidades de glóbulos rojos y fue trasladada a la Unidad de Cuidados Intensivos (UCI) con intubación orotraqueal, para soporte ventilatorio e inotrópico.

En UCI, la paciente permaneció inestable hemodinámicamente. A partir de los exámenes paraclínicos de control se evidenció que el nivel de hemoglobina era 2,8 g/dL, por lo que se le realizó transfusión de 6 unidades de glóbulos rojos y fue llevada a laparotomía exploratoria; procedimiento en el cual se encontró hemoperitoneo de 2.000 cc, el cual fue drenado. Seguidamente, fue empaquetada con 3 compresas a nivel de cúpula vaginal y se le colocó una bolsa de Viaflex ${ }^{\circledR}$. Veinticuatro horas después, se procedió a llevar a la paciente a lavado peritoneal para desempaquetamiento y cierre de la pared abdominal. Luego de permanecer once 
días en la UCI, la paciente fue trasladada al piso de hospitalización de ginecología y obstetricia.

Durante su primer día en el área de hospitalización general, la paciente refirió sensación de dificultad respiratoria acompañada de picos febriles, taquipnea e hipoventilación bibasal. Enseguida, se le tomó una radiografía de tórax que mostró atelectasia basal derecha e imágenes compatibles con proceso de consolidación en el lóbulo inferior izquierdo. A su vez, el hemograma reveló 10.900 leucocitos, 85\% de neutrófilos y 9,5 de hemoglobina, a partir de lo cual se le diagnosticó neumonía nosocomial y se inició tratamiento con antibióticos y oxigenoterapia.

Sin embargo, pese al tratamiento instaurado, la paciente presentó deterioro clínico consistente en persistencia de picos febriles, intolerancia a la vía oral, taquicardia, distensión e hipertimpanismo abdominal con leve dolor en hemiabdomen derecho y peristaltismo disminuido. Por tal motivo, se solicitó una radiografía de abdomen, la cual evidenció un cuadro obstructivo muy probablemente a nivel del intestino delgado (figura 1). Inmediatamente después, fue valorada por el servicio de cirugía general,

Figura 1. Dilatación del colon, especialmente del transverso.

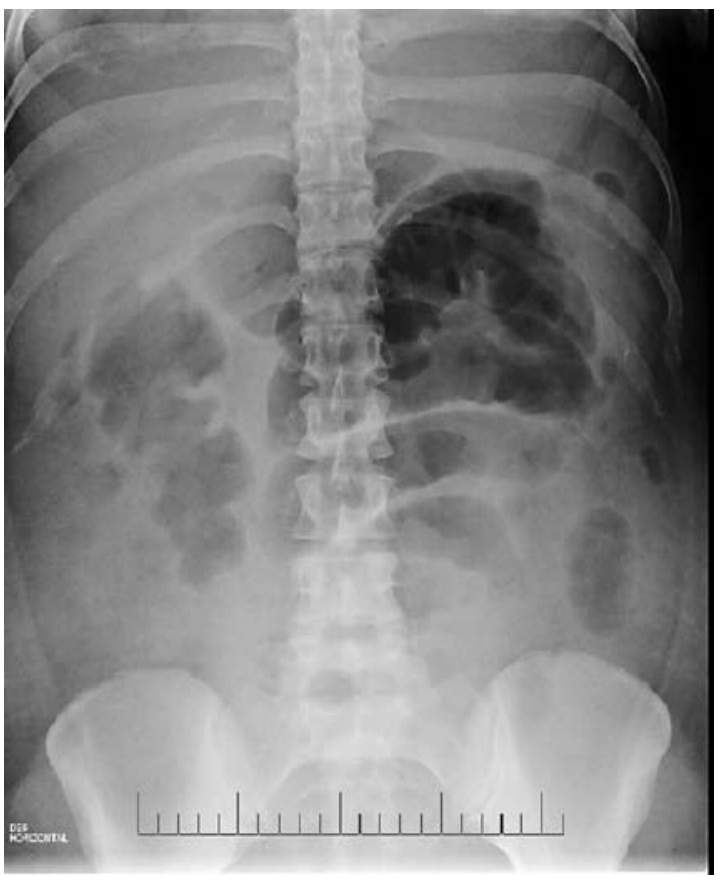

quienes decidieron llevarle a cabo una laparotomía exploratoria donde se encontraron con una aponeurosis de mala calidad, una evisceración contenida, una peritonitis fecal de 500 cc, una perforación colónica de $0,5 \mathrm{~cm}$ a nivel de la válvula ileocecal y una distensión de asas delgadas sin evidencia de obstrucción mecánica; por lo cual se le realizó drenaje de peritonitis fecal, lavado peritoneal e ileostomía en asa de protección.

Posteriormente, la paciente fue trasladada a la sala de recuperación con diagnóstico de síndrome de Ogilvie donde permaneció hospitalizada catorce días más, presentando una evolución clínica satisfactoria por lo que finalmente se le dio de alta.

\section{METODOLOGÍA}

La búsqueda de la información se realizó en las bases de datos SciELO, LILACS, Redalyc, IMBIOMED y PubMed con la terminología MeSH "Ogilvie syndrome", "hysterectomy", "caesarean section", empleando "ADN" como conector boleano. Bajo estas características, se encontraron 52 artículos, de los cuales se seleccionaron en primera instancia aquellos que estaban en inglés y hacían referencia, en el título del artículo o en el resumen, al SO después de procedimientos gineco-obstétricos. En total, se obtuvieron 14 reportes de caso, 2 series de caso, 3 artículos de revisión; al igual que 7 artículos de investigación y 6 artículos de revisión que hacían referencia al tratamiento de esta entidad y finalmente, 3 libros de texto.

\section{DISCUSIÓN}

Epidemiología: la frecuencia exacta de esta entidad todavía no ha sido establecida, ya que es una condición clínica rara que ocurre en $0,1 \%$ de los pacientes sometidos a cirugía, en 0,05\% de los pacientes con trauma y en $0,3 \%$ de los pacientes críticamente enfermos con quemaduras. ${ }^{3}$ Asimismo, algunos investigadores sugieren que esta enfermedad es más común en los adultos mayores ${ }^{4}$ y puede tener un ligero predominio masculino, posiblemente en una proporción de 1,5:1. ${ }^{5}$ 
Por su parte, en mujeres delgadas, saludables y en edad reproductiva, la cesárea es el procedimiento quirúrgico más frecuentemente asociado con este síndrome, ${ }^{6-11}$ aunque también se han presentado casos después de un parto vaginal, ${ }^{9}$ un parto instrumentado ${ }^{6}$ una cesárea - histerectomía, ${ }^{12}$ una histerectomía, ${ }^{13,14}$ y un embarazo con parto prematuro, preeclampsia y embarazos gemelares. ${ }^{11,15-17}$ Rieger y sus colegas ${ }^{16}$ han reportado casos de este síndrome durante el embarazo, que manejados conservadoramente, han concluido de manera favorable tanto para la madre como para el feto.

Pese a esto, las tasas de mortalidad varían de $12 \%$ a 50\%, 12\%-30\% de los casos sin perforación y 40\%-50\% de los casos con perforación. ${ }^{4,18}$ En el Reino Unido se ha reportado que alrededor de 20 mujeres mueren al año por esta entidad. ${ }^{5,19}$ Por ejemplo, el Confidential Enquiry into Maternal and Child Health Report (CEMACH) de Londres informó de cuatro muertes a causa del síndrome de Ogilvie desde 2000 a 2002, todos ocurridos después de procedimientos de cesárea. ${ }^{20}$

Fisiopatología: aunque aún se desconoce la causa exacta de dicha enfermedad, se ha descrito que a ésta podría subyacer un desbalance entre la inervación simpática y parasimpática del colon. Vanek y colegas, en una serie de caso, sugieren que este síndrome puede deberse a una neuroapraxia de los nervios parasimpáticos del sacro (S2, S3, S4). ${ }^{5}$ Estos nervios pasan debajo del plexo hipogástrico en estrecha proximidad con el cuello del útero, de la vagina, de los ligamentos anchos ${ }^{11} \mathrm{y}$ terminan inervando el colon izquierdo y el recto siendo éstos los responsables del vaciamiento colónico, lo cual puede explicar la asociación entre los procedimientos gineco-obstétricos y el síndrome de Ogilvie. ${ }^{7}$ Igualmente, es importante tener en cuenta las situaciones que pueden alterar la regulación autonómica del colon, ya sea por supresión parasimpática o por excesiva estimulación simpática, ${ }^{3}$ entre las cuales se encuentran: antecedentes quirúrgicos y traumáticos (cirugía pélvica por trauma, transplante renal, cirugía de cadera, fractura femoral, injuria retroperitoneal, ventilación mecánica); situaciones médicas severas previas, antecedentes farmacológicos (opiáceos, narcóticos, fenotizidas, polisterine sódico, enemas de sulfonato y sorbitol, antidepresivos triciclitos); y factores neoplásicos (tumores retroperitoneales, leucemia, radioterapia pélvica, coriocarcinoma). ${ }^{21}$ También se han mencionado otros factores como posibles causas. Bachulis y Smith, por ejemplo, estudiaron una serie de 35 casos encontrando una función anormal de las prostaglandinas. ${ }^{22}$ Del mismo modo, otros datos recientes indican que la volatilidad gaseosa corta y el óxido nítrico (NO, por sus siglas en inglés), que son producidos en exceso en algunas patologías, pueden generar fenómenos de dismotilidad y dilatación. ${ }^{23}$

Es por esto que, como resultado de la causa subyacente, ocurre una depleción del volumen debido a que el contenido alimentario es atrapado en las dilataciones del colon pseudoobstruido, que a su vez se asocia con pérdida de agua a través del vómito y del secuestro de líquidos en la cavidad abdominal por transudado, lo cual lleva a un desequilibrio hidroelectrolítico. ${ }^{24}$

En la fisiopatología, el ciego juega un papel muy importante ya que es la parte del colon con mayor diámetro, y una mínima cantidad de presión conduce a un aumento en su tamaño y por lo tanto puede dilatarse más rápidamente que el resto del colon. Debido a esto, es más propenso a una ruptura, especialmente entre la serosa de las tenias. ${ }^{7}$ Estudios retrospectivos en animales sugieren un diámetro intracecal de $12 \mathrm{~cm}$ como umbral crítico para la ruptura del ciego, ${ }^{25} \mathrm{y}$ existen datos que prueban que en presencia de una presión mayor a $26 \mathrm{~cm} \mathrm{H}_{2} \mathrm{O}$ se corre el riesgo de necrosis isquémica y perforación. ${ }^{26}$ Esto es importante para la toma de decisiones en cuanto a la conducta quirúrgica a seguir, ya que significa que un diámetro cecal de $9 \mathrm{~cm}$ a $12 \mathrm{~cm}$ permite un tratamiento conservador, mientras que un diámetro mayor de $12 \mathrm{~cm}$ sin respuesta a manejo conservador después de 48 horas indica que se debe realizar una intervención quirúrgica. ${ }^{12}$ 


\section{Diagnóstico}

Frecuentemente, los síntomas se producen durante los 2 a 12 días posteriores al procedimiento quirúrgico. Los signos son similares a aquellos de una gran obstrucción mecánica intestinal; 80\% de las mujeres presenta dolor abdominal mientras que en 90\%-100\% de los casos se observa distensión abdominal. ${ }^{2}$ Asimismo, hay disminución o cesación de movimientos intestinales en $40 \%$ de los casos, salida escasa de materia fecal o flatos, náuseas y vómitos de aparición tardía; y se debe sospechar sepsis, necrosis o perforación intestinal si se presenta fiebre. Además, es posible encontrar a la paciente en un estado agudo de deshidratación, de oliguria y de desequilibrio hidroelectrolítico, incluso algunas de ellas desarrollan alteraciones renales y hepáticas. ${ }^{23}$ Finalmente, la presencia de dolor localizado en la fosa ilíaca derecha indica una inminencia de ruptura del ciego. ${ }^{26}$

Ante esto, los diagnósticos diferenciales que se deben tener en cuenta son: obstrucción mecánica, vólvulos, peritonitis, perforación intestinal y sangrado intraabdominal o retroperitoneal. ${ }^{24}$

Entre los métodos de diagnóstico, la radiografía de abdomen simple es el procedimiento más útil para detectar la dilatación intestinal, especialmente la del ciego mientras que la presencia de pneumoperitoneo confirma la presencia de una perforación intestinal. ${ }^{24}$ Del mismo modo, también es factible utilizar un medio de contraste hidrosoluble con el fin de descartar una obstrucción mecánica, y en el caso del Síndrome de Ogilvie habría un buen tránsito del medio de contraste hasta el ciego. ${ }^{27}$

\section{Manejo}

Terapia conservadora: en las dilataciones de un diámetro cecal menor de $12 \mathrm{~cm}$, se utiliza un tratamiento conservador; de modo que se debe suspender la vía oral, y colocar una sonda nasogástrica para descompresión. En caso de existir algún desequilibrio hidroelectrolítico, se deben tomar las medidas pertinentes para su corrección. ${ }^{25}$
Igualmente, es importante optimizar el aporte de líquidos intravenosos y la monitorización hemodinámica del paciente, como también realizar una revisión de los medicamentos que recibe y que puedan afectar la motilidad colónica. También se pueden llevar a cabo cuidadosos exámenes rectales intermitentes, evitar la posición supina y elevar el recto posicionando al paciente con almohadas bajo la pelvis ${ }^{24}$ con el fin de facilitar la expulsión de flatos.

Si la paciente aún esta embarazada, la condición fetal deberá ser estrictamente monitorizada. La terapia conservadora puede ser usada en las primeras 24 a 48 horas antes de emplear una terapia farmacológica o una intervención endoscópica. Las tasas de éxito de la terapia conservadora varían de $20 \%$ a $92 \% .^{2}$

Terapia farmacológica: el único medicamento que ha reportado beneficios es la neostigmina. ${ }^{27-34}$ Este fármaco, actúa inhibiendo la acetilcolinesterasa y, de esta manera, restaura el balance autonómico. El tratamiento con neostigmina consiste en una inyección intravenosa de 2,5 mg administrados en un período de 3 a 5 minutos, pero sólo 30 minutos después de la aplicación el paciente comienza a percibir una respuesta.

Terapia endoscópica: la descompresión por colonoscopia fue utilizada por primera vez en 1977; esta técnica quirúrgica se ha realizado con éxito en varios cientos de pacientes que no responden a la terapia farmacológica con neostigmina. Pese a que su eficacia no ha sido establecida aún en ensayos clínicos aleatorizados, existen reportes que indican que mediante esta técnica se logra una tasa de éxito en $61 \%$ a $78 \%$ de los casos; ${ }^{25}$ aunque la pseudoobstrucción puede recurrir hasta en 40\% de los mismos. ${ }^{34}$

Cirugía abierta: la cirugía es la conducta de elección cuando existe sospecha de isquemia o perforación intestinal. Generalmente se realiza una resección intestinal con derivación fecal temporal, por lo que es necesaria una segunda etapa para el cierre definitivo. ${ }^{21}$ 


\section{CONCLUSIÓN}

El Síndrome de Ogilvie es una patología poco frecuente que se puede presentar como complicación de algunas cirugías gineco-obstétricas.

Es importante tener en cuenta esta patología como diagnóstico diferencial en estas pacientes en caso de presentar distensión abdominal durante su evolución posoperatoria, ya que el diagnóstico temprano disminuye la necesidad de tratamiento quirúrgico y la morbimortalidad gracias a la posibilidad de instaurar un manejo conservador o farmacológico.

\section{AGRADECIMIENTOS}

A la Doctora Margarita Quijano Serrano por la ayuda en la preparación de este manuscrito y en la revisión de este caso.

\section{REFERENCIAS}

1. Ogilvie H. Large-intestine colic due to sympathetic deprivation: a new clinical syndrome. Br Med J 1948;2:671-3.

2. Carpenter S, Holmstorm B. Ogilvie Syndrome. Visitado en 2009 Ago 12. Disponible en: http://www. eMedicine.com/ med/topic2699.htm

3. Kadesky K, Purdue GF, Hunt JL. Acute pseudoobstruction in critically ill patients with burns. J Burn Care Rehabil 1995;6:132-5.

4. Srivastava G, Pilkington A, Nallala D, Polson DW, Holt E. Ogilvie's syndrome: a case report. Arch Gynecol Obstet 2007;276:555-7.

5. Munro A. Large bowel obstruction. En: Wills BW, PatersonBrown S, editors. Hamilton Bailey's Emergency Surgery. 13th ed. London: Hodder Arnold; 2000. p. 436-39.

6. Roberts CA. Ogilvie's syndrome after cesarean delivery. J Obstet Gynecol Neonatal Nurs 2000;29:239-46.

7. Vanek VW, Al-Salti M. Acute pseudo-obstruction of the colon (Ogilvie's syndrome). An analysis of 400 cases. Dis Colon Rectum 1986;29:203-10.

8. Weber P, Heckel S, Hummel M, Dellenbach P. Ogilvie's syndrome after cesarean section. Apropos of 3 cases. Review of the literature. J Gynecol Obstet Biol Reprod (Paris) 1993;22:653-8.

9. Moore JG, Gladstone NS, Lucas GW, Ravry MJ, Ansari AH. Successful management of post-cesarean-section acute pseudoobstruction of the colon (Ogilvie's syndrome) with colonoscopic decompression. A case report. J Reprod Med 1986;31:1001-4.

10. Rodríguez-Ballesteros R, Torres-Bautista A, TorresValadez F, Ruiz-Moreno JA. Ogilvie's syndrome in the postcesarean section patient. Int J Gynecol Obstet 1989;28:185-7.

11. Nanni G, Garbini A, Luchetti P, Nanni G, Ronconi P, Castagneto M. Ogilvie's syndrome (acute colonic pseudo-obstruction): review of the literature (October 1948 to March 1980) and report of four additional cases. Dis Colon Rectum 1982;25:157-66.

12. De M, Mandal A, Cooper JC. A case of Ogilvie's syndrome after caesarean section. J Obstet Gynaecol 2002;22:686-7.

13. Kakarla A, Posnett H, Jain A, Ash A. Acute pseudoobstruction of the colon (Ogilvie's syndrome) following instrumental vaginal delivery: case report. Int J Clin Pract 2006;60:1303-5.

14. Hamed AD, Dare FO. Ogilvie's syndrome. Int J Gynaecol Obstet 1992;37:47-50.

15. Singh P, Ilancheran A, Ti TK, Ratnam SS. Ogilvie's syndrome of colonic pseudo-obstruction: a complication of radical hysterectomy with pelvic and paraaortic lymphadenectomy. Gynecol Oncol 1989;32:390-3.

16. Schreiner B. Ogilvie's syndrome as a complication following extensive total abdominal hysterectomy. Schweiz Med Wochenschr 1988;118:726-8.

17. Pecha RE, Danilewitz M. Acute pseudo-obstruction of the colon (Ogilvie's syndrome) resulting from combination tocolytic therapy. Am J Gastroenterol 1996;91:1265-6.

18. Rieger NA, Lyon JW, Bryce RL, Birrell SN. A case of acute colonic pseudo-obstruction in pregnancy. Aust N ZJ Obstet Gynaecol 1996;36:363-5.

19. Kalu E, Fakokunde A, Jesudason M, Whitlow B. Acute colonic pseudo-obstruction (Ogilvie's syndrome) following caesarean section for triplets. J Obstet Gynaecol 2005;25:299-300.

20. Lewis G, Drife J, editors. Why Mothers Die 20002002. Confidential Enquiry into Maternal and Child Health. London: RCOG Press; 2004. p. 232-33.

21. Bachulis BL, Smith PE. Pseudo-obstruction of the colon. Am J Surg 1978;136:66-72.

22. De Giorgio R, Barbara G, Stanghellini V, Tonini M, Vasina V, Cola B, et al. Review article: the pharmacological treatment of acute colonic pseudo-obstruction. Aliment Pharmacol Ther 2001;15:1717-27. 
23. Mourelle M, Casellas F, Guarner F, Salas A, Riveros-Moreno $\mathrm{V}$, Moncada S, et al. Induction of nitric oxide synthase in colonic smooth muscle from patients with toxic megacolon. Gastroenterology 1995;109:1497-502.

24. Kakarla A, Posnett H, Jain A, George M, Ash A. Review Acute colonic pseudo-obstruction after caesarean section. The Obstetrician \& Gynaecologist 2006;8:207-13.

25. Eisen GM, Baron TH, Dominitz JA, Faigel DO, Goldstein JL, Johanson JF, et al. Acute colonic pseudoobstruction. Gastrointest Endosc 2002;56:789-92.

26. Munro A. Large bowel obstruction. En: Wills BW, Paterson-Brown S, editors. Hamilton Bailey's Emergency Surgery. 13th ed. London: Hodder Arnold; 2000. p. 436-39.

27. Hutchinson R, Griffiths C. Acute colonic pseudoobstruction: a pharmacological approach. Ann R Coll Surg Eng 1992;74:364-7.

28. Stephenson BM, Morgan AR, Salaman JR, Wheeler MH. Ogilvie's syndrome: a new approach to an old problem. Dis Colon Rectum 1995;38:424-7.
29. Turégano-Fuentes F, Muñoz-Jiménez F, Del ValleHernández E, Pérez- Díaz D, Calvo-Serrano M, et al. Early resolution of Ogilvie's syndrome with intravenous neostigmine: a simple effective treatment. Dis Colon Rectum 1997;40:1353-7.

30. Paran H, Silverberg D, Mayo A, Shwartz I, Neufeld D, Freund U. Treatment of acute colonic pseudoobstruction with neostigmine. J Am Coll Surg 2000;190:315-8.

31. Ponec RJ, Saunders MD, Kimmey MB. Neostigmine for the treatment of acute colonic pseudo-obstruction. N Eng J Med 1999;341:137-41.

32. Trevisani GT, Hyman NH, Church JM. Neostigmine: safe and effective treatment for acute colonic pseudoobstruction. Dis Colon Rectum 2000;43:599-603.

33. Abeyta BJ, Albrecht RM, Schermer CR. Retrospective study of neostigmine for the treatment of acute colonic pseudo-obstruction. Am Surg 2001;67:265-8.

34. Rex DK. Colonoscopy and acute colonic pseudoobstruction. Gastrointest Endosc Clin N Am 1997;7:499-508. 